\title{
Simulation of a vibrating reed exciter for thickening fabrics in the weaving loom
}

\author{
M. Kuchar*, K. Siczek** \\ *Lodz University of Technology, Zeromskiego Str. 116, 90-924 Lodz, Poland, E-mail: maciej.kuchar@p.lodz.pl \\ **Lodz University of Technology, Zeromskiego Str. 116, 90-924 Lodz, Poland, E-mail: ks670907@p.lodz.pl \\ cross $^{\text {ref }}$ http://dx.doi.org/10.5755/j01.mech.22.5.13360
}

\section{Introduction}

To improve the conditions of thickening threads during the weaving process the reed was set into vibration. The additional vibrating movement of the reed has significantly lower amplitude and higher frequency comparing to the basic movement of the reed resulting from the movement of the slay [1]. The vibrating movement of the reed during the thickening process has a positive impact on reducing the dynamic loading of the warp and on the obtained density of threads in the produced fabric. The vibrating system of the reed must operate under appropriate frequency and amplitude. Figure 1 shows a slay vibrating mechanism. The mechanism is based on the bending ability of the reed blades 1 in the range of elastic strains. The vibrating movement of the read is induced by an electromagnetic exciter 2 [2]. The reed and the exciter are fixed on the slay 3 .

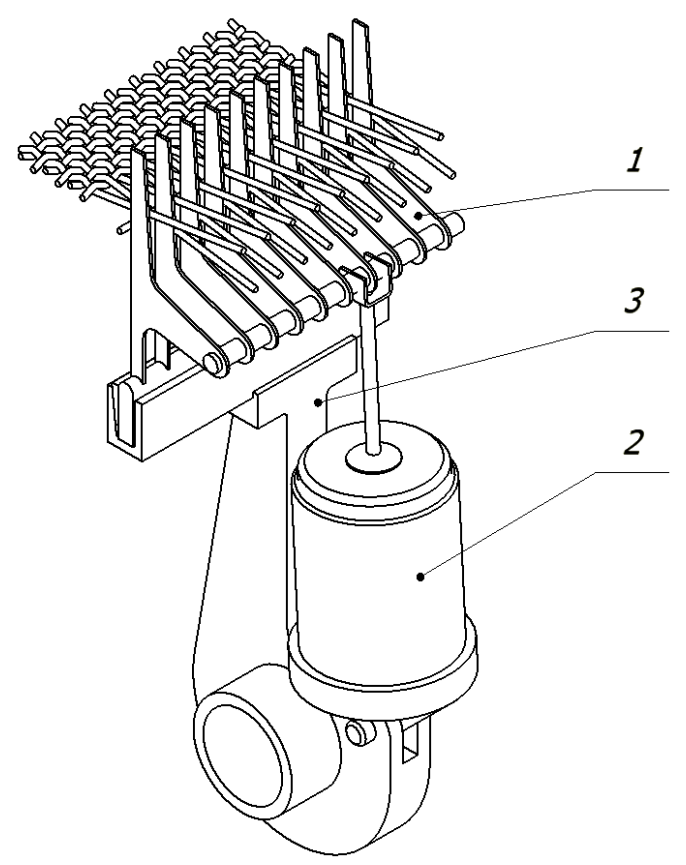

Fig. 1 The vibrating slay mechanism

The cross-section of the exciter is shown in Fig. 2. The exciter consists of a movable element- a pusher 5 linked to the carcass with some coils 3 (copper wire with a diameter of $0,5 \mathrm{~mm}$ ) and a stator which includes: a neodymium permanent magnet 1 , pole pieces 2 , and a clamping sleeve 4 . The terminals of the exciter are supplied with alternating voltage. The aim of study is to evaluate the operation of the exciter in the vibrating slay mechanism. The evaluated parameter is the amplitude of the vibrating movement of the reed during thickening of typical fabrics. Current intensity in the exciter coils is also an important parameter. In order to analyse the exciter, it is necessary to adopt an appropriate model of the vibrating system and to determine its electric and dynamic parameters. During the analysis a crucial parameter is the inductance of the pusher coils in the predicted range of their motion. The change of stiffness and damping coeficient of the vibrating system during the thickening cycle, which is related to the phase of contact between the reed and the fabric fell are also of great significance.

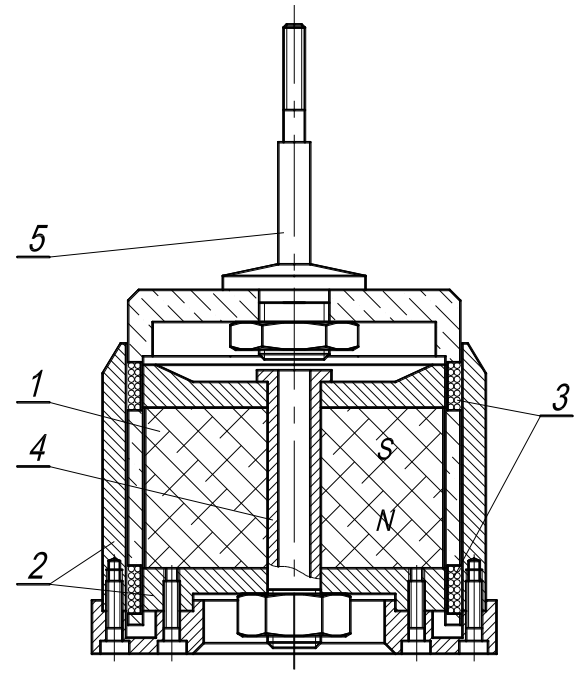

Fig. 2 Electromagnetic exciter of reed vibrations

\section{Magnetic field distribution in the exciter}

Distribution of the magnetic field in the magnetic circuit of the exciter consisting of a core, two pole pieces, a permanent magnet and some air gaps, is obtained on the basis of the finite element method (FEM). In the air gaps of the magnetic circuit there are some serially connected coil segments, with counter-wound windings. Alternating current flows in the electrical circuit, which includes copper windings of the coil segments, generating the resultant electro-dynamic force, which sets the coil segments into motion along their axis.

During the analysis, eddy currents were disregarded but consideration was given to the axial symmetry in the analyzed tubular motor (exciter) with permanent magnets. Linear magnetization of the materials was assumed. In the iterative process of calculations, the nonlinearity of magnetization of ferromagnetic materials was also taken into account. 
It was assumed that the material characteristics of the core and the pole pieces correspond to low-carbon steel [3], and the demagnetization curve of the permanent magnet corresponds to this of the Nd-Fe-B magnet [3]. The cross-section of the core, the pole pieces, permanent magnets, coils, and air segments are shown in Fig. 3.

The zero Dirichlet condition (1) is fulfilled on the axis of symmetry [3]- Fig. 3:

$$
A_{z}=0 \text {, }
$$

and outside of the area of calculations mixed boundary condition was set (2) - Fig. 3:

$$
\frac{1}{\mu_{0} \mu_{r}} \frac{\partial A}{\partial n}+b_{0} A+b_{1}=0,
$$

where $\frac{\partial A}{\partial n}$ is the normal derivative outside the border of the area of calculations.

The values of the coefficients $b_{0}$ and $b_{1}$ are determined in an iterative process based on an algorithm used by the FEMM program. Adopting the mixed boundary condition accurately reflects the unlimited field distribution around the area of the tubular engine.

The mesh of triangular finite elements was generated by the FEMM program. The degrees of freedom for the nodes are the values of the component $A_{z}$ of the vector magnetic potential

Some examples of the distribution of magnetic field lines (tangent to the vector magnetic potential A) and magnetic field induction B are shown in Fig. 4. There was no area of magnetic saturation, because the calculated value of the magnetic field density did not exceed magnetic saturation value for steel, which is about $2.2 \mathrm{~T}$.

The inductance of the exciter coil was calculated by FEMM program.

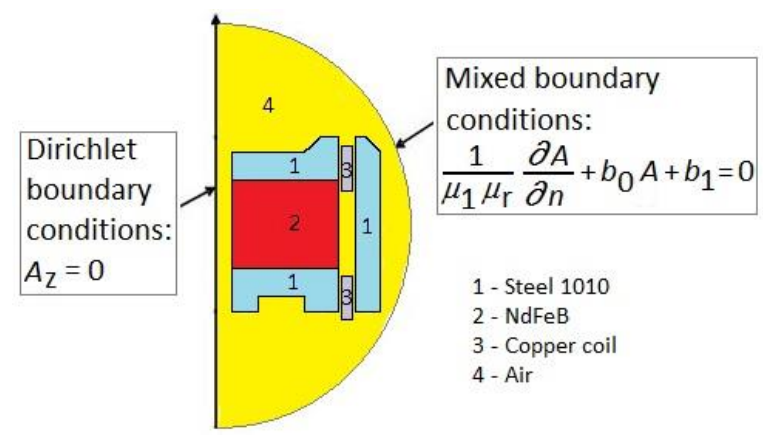

Fig. 3 Regions with different material properties, boundary conditions for the model of an electromagnetic drive

The obtained waveforms of the inductance against displacement of the coil segments relative to the stator along the $y$-axis for different values of current flowing in the coil winding are presented in Fig. 5. It can be seen that the waveforms of the inductance are almost independent of the displacement, for displacements smaller than $1 \mathrm{~mm}$. For values higher than $1 \mathrm{~mm}$, the coil inductance slightly decreases. Also the increase of current flowing in the coil winding results in only slight decrease of the coil inductance. Therefore, for further analysis, it can be assumed, that the value of the coil inductance is constant.

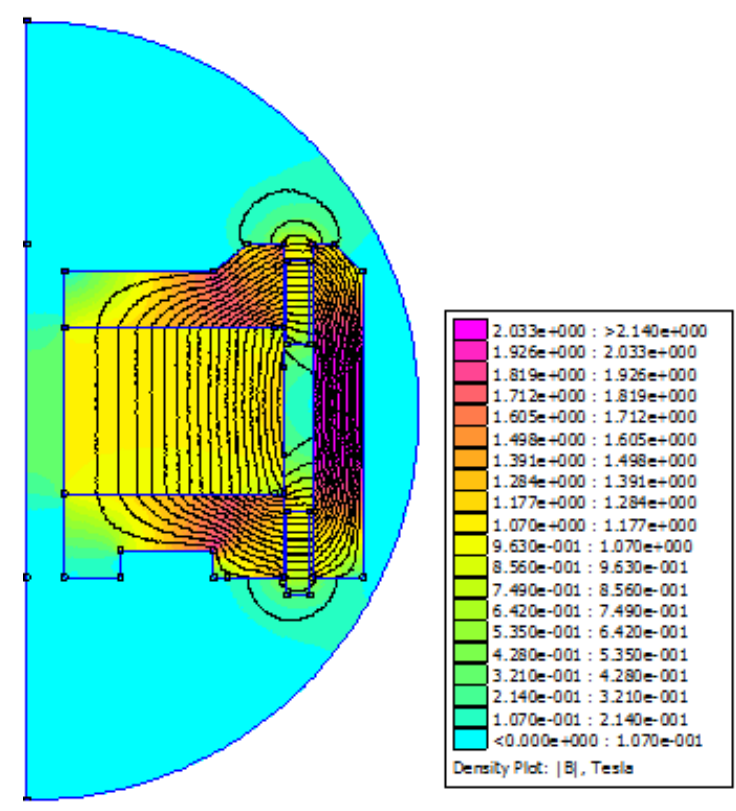

Fig. 4 Distribution of the magnetic field lines (tangent to the vector magnetic potential $A$ ), magnetic field density $B$

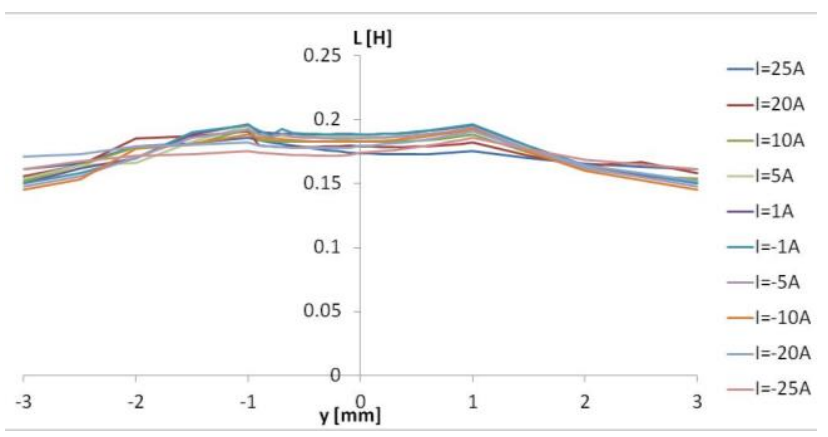

Fig. 5 Waveforms of the inductance against displacement of coil segments relative to the stator along the $y$-axis for different values of current intensity in coil

\section{Simulation model of the movement of the vibrating system}

The movement of the reed at the fabric fell results from the basic movement of the slay $x$ and the overlaping vibrating motion. The shift of the pusher y relative to the stator along the axis of the exciter is proportional to the vibrating motion. The amplitude of the latter movement at the fell of the fabric is close to the amplitude of the pusher movement. The stator of the exciter is connected to the slay. In order to carry out the analysis, a dynamic model of the system shown in Fig. 6 was adopted.

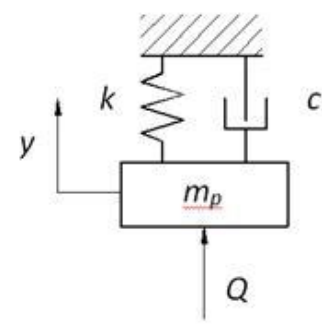

Fig. 6 Dynamic model of the vibrating system

The exciting force generated by the exciter $Q$ 
causes vibrating movement of the pusher relative to the slay which is described by the Eq. (3):

$$
Q(t)=\ddot{y} m_{p}+\dot{y} c+y k,
$$

where $k$ is the stiffness and $c$ is damping coefficient of the system, reduced to the axis of the exciter.

Fig. 7 presents stiffness $k$ and damping coefficient $c$ of the vibrating system as a function of the reed movement y measured at the fabric fell. The values of these parameters and the reed movement are presented as relative and referred to their peak values.

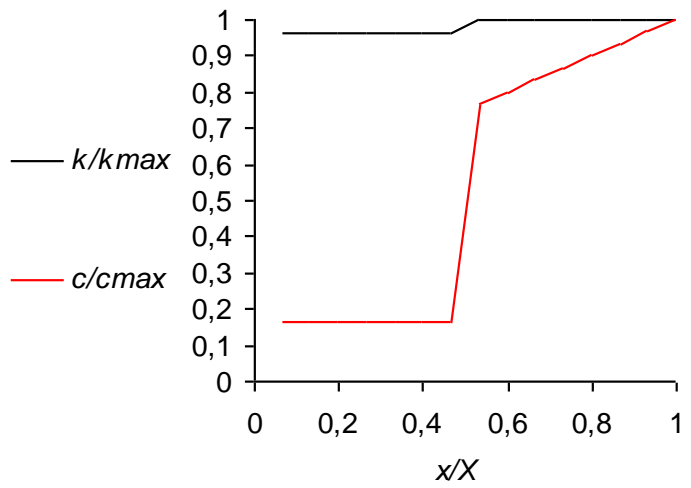

Fig. 7 Stiffness and damping coefficient of the system against the reed movement

Equivalent stiffness of the system $k_{w}$, reduced to the pusher axis, results mainly from the stiffness of the reed and together with the sum of masses: the replacing mass of the reed and the movable element of the exciter $m_{\mathrm{p}}$ determines resonant frequency of the system $f_{w}$. During the contact between the reed and the fabric fell, additional stiffness results from the fabric - warp system $k_{t}[4,5]$. Generally, this stiffness is small in relation to the stiffness of the reed. The value of damping coefficient $c_{w}$, reduced to the axis of the pusher in the initial phase of the cycle is mainly caused by the resistance to motion of the exciter [6]. During the contact between the reed and the fabric fell there is a sharp increase in the value of the damping coefficient $[7,8]$. Later in the cycle, the increasing value of the damping coefficient $c_{t}$ results from the process of thicken ing the threads and its progressive nature has been identified experimentally [8]. Therefore, stiffness $k$ and damping coefficient $c$ can be described by the Eqs. (4) and (5), respectively.

$$
\begin{aligned}
& k=k_{w}+k_{t} ; \\
& c=c_{w}+c_{t} .
\end{aligned}
$$

The assumed values of stiffness $k_{t}=40 \mathrm{kN} / \mathrm{m}$ and peak values of damping coefficient in the range of $c_{t}=20-30 \mathrm{Ns} / \mathrm{m}$ correspond to obtaining relatively large thickening of fabrics with typical dynamic parameters (e.g wefts and warps made of cotton yarn 30-60tex, with thread diameter below 1 $\mathrm{mm}$ ) [9-14]. A slight increase of stiffness and high increase of the damping coefficient, related to the phase of contact between the reed and the fabric fell, reduce the operating amplitude of the vibrating motion.

Assuming constant inductance $L$, and adopting $C_{e}$ as an electric constant of the exciter, the sum of voltage drops in the coils circuit was described by the Eq. (6) [11]:

$$
\dot{y} C_{e}+L \frac{d i}{d t}+R i=u(t) .
$$

The exciting force was determined by the Eq. (7)

[12]:

$$
Q(t)=C_{e} i
$$

Based on the dependences (3)-(7) it was possible to calculate the values of the basic dynamic and electric parameters for the vibrating reed system during the thickening cycle. The calculations refer to the section of the reed equal to $0,2 \mathrm{~m}$ which is assumed to be induced by one of the exciters placed symmetrically across the width of the reed. The movement character of the slay corresponds to the sine function in the range of $0-0,5 \pi$ with the amplitude of $X=20 \mathrm{~mm}$ measured at the fell of the fabric in extreme position of the slay. Additionally, the following parameters were adopted for calculations: $m_{p}=0,2 \mathrm{~kg} ; c_{w}=3 \mathrm{Ns} / \mathrm{m}$; $k_{w}=1400 \mathrm{kN} / \mathrm{m} ; f_{w}=400 \mathrm{~Hz} ; k_{t}=0-80 \mathrm{k} \mathrm{N} / \mathrm{m} ; L=200 \mu \mathrm{H}$; $C_{e}=5 \mathrm{~N} / \mathrm{A} ; u= \pm 6-15 \mathrm{~V}$.

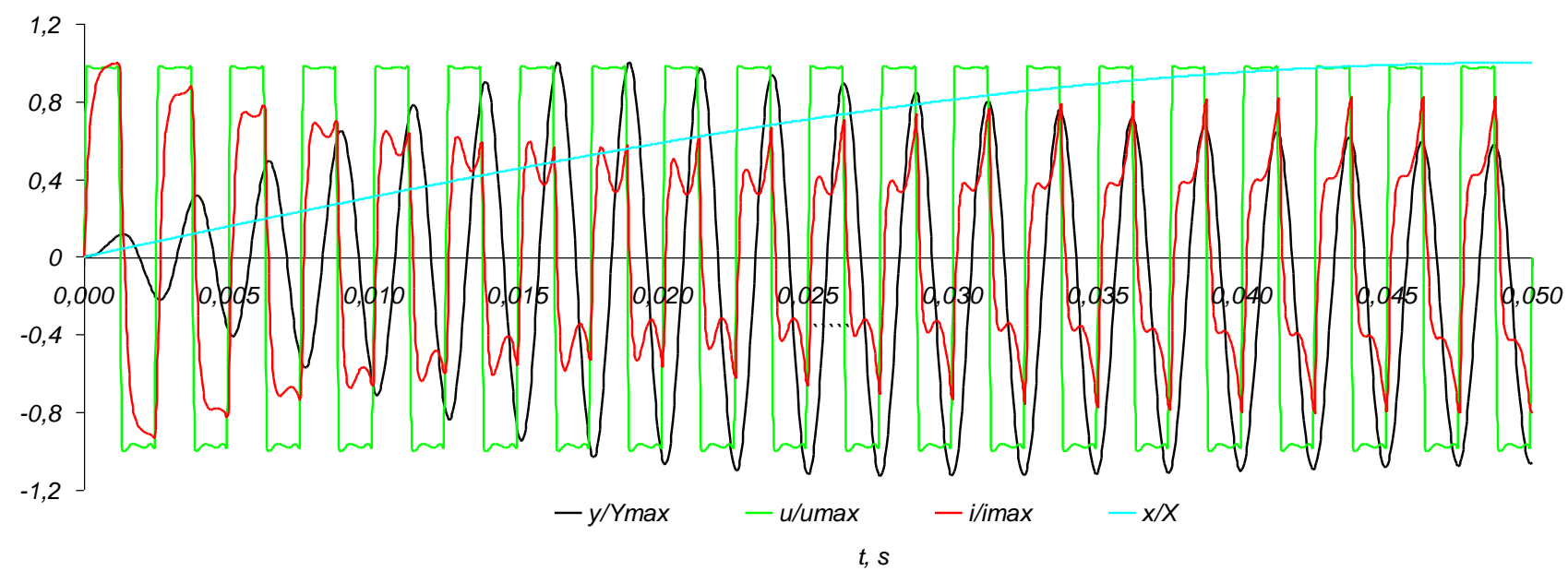

Fig. 8 Relative waveforms of: voltage, current intensity and displacement of the pusher $\left(u= \pm 10 \mathrm{~V} ; k_{t}=40 \mathrm{kN} / \mathrm{m}\right.$; $c_{t}=22 \mathrm{Ns} / \mathrm{m} ; Y_{\max }=0,6 \mathrm{~mm} ; i_{\max }=5 \mathrm{~A} ; X=20 \mathrm{~mm}$ ) 
Simulations of the time-varying dynamic and electric parameters of the system were made using the INVENTOR 2009 program. Fig. 8 shows the waveforms of the reed movement resulting from the movement of the slay measured at the fell of the fabric, voltage supplying the coils, current intensity and pusher vibrating motion relative to the slay. The waveforms are shown as relative and referred to their peak value.

The amplitude of the pusher vibrating motion $Y$ increases in the initial phase of the start-up, reaching its peak value $Y_{\max }$, and then, in the phase of the contact between the reed and the fabric fell, it drops, reaching its lowest operating value $Y_{0}$ in the extreme front position of the slay. Regardless of that, the course of motion deviates due to the increasing force resulting from the impact of the fabric - warp system, loading the slay during thickening $[1,4,5,7,8]$. These changes increase with the stiffness of the fabric - warp system. Within the range of a single movement of the pusher, current intensity in the coils deviates from the relatively constant supply voltage. Current waveform is similar to the symmetrical one occurring in the initial phase of the start-up. Then, during the contact phase between the reed and the fabric fell the waveform undergoes one-sided deflection. In that phase apart from the beginning of the start-up phase, peak values of current intensity can be observed.

\section{Results of simulation studies}

For effective vibrational thickening of the fabrics, it is necessary to obtain the amplitude of the reed motion of at least $30-50 \%$ of the thread diameter $[4,7,8]$. Figure 9 presents the waveforms of the vibrating motion of the pusher in the conditions of increasing stiffness of the fabric - warp system. The resulting amplitudes of the pusher movement are sufficient for effective vibrational thickening of threads in fabrics with typical dynamic parameters. Differences in the waveforms emerge in the phase of the contact between the reed and the fabric fell. The decrease in the amplitude of the pusher movement occurs due to additional stiffness originating from the fabric - warp system, influencing the change in its resonant frequency.

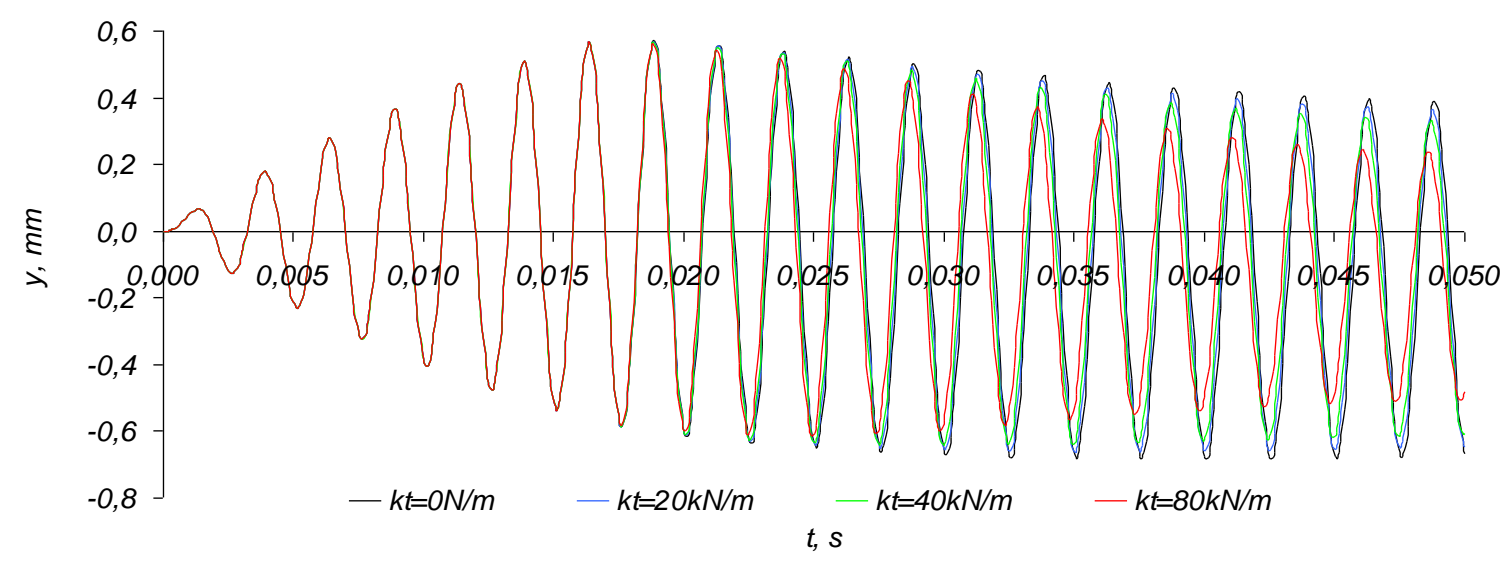

Fig. 9 Movement of the pusher under different stiffness of the fabric-warp system $\left(u= \pm 10 \mathrm{~V} ; c_{t}=22 \mathrm{Ns} / \mathrm{m}\right)$

Fig. 10 shows the waveforms of the current intensity in the exciter coils under the condition of increasing stiffness of the fabric - warp system. The range of the waveforms includes the phase of the contact between the reed and the fabric fell. Both, the unilateral deviation from symmetrical course in a single movement of the pusher and the peak current intensity increase with the stiffness.

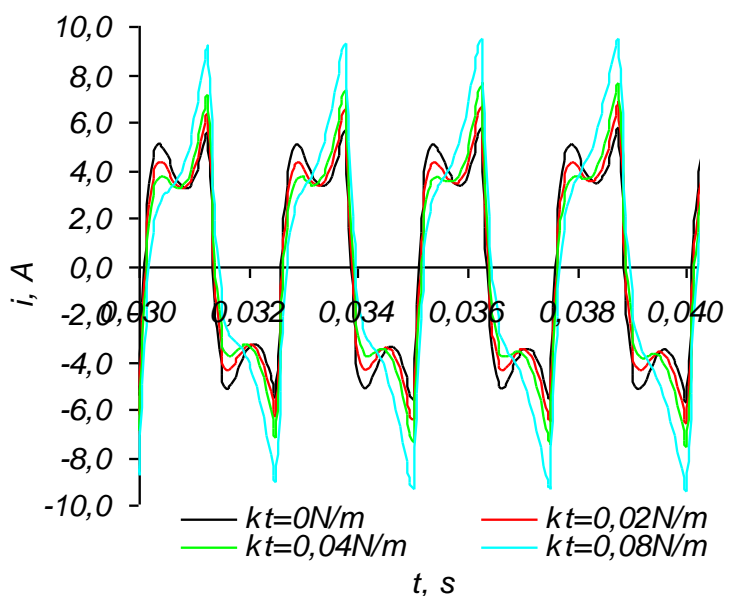

Fig. 10 Waveforms of current intensity against time for different stiffness of the fabric-warp system $\left(u= \pm 10 \mathrm{~V} ; c_{t}=22 \mathrm{Ns} / \mathrm{m}\right)$
Fig. 11 shows the waveforms of the current intensity in the exciter coils under the condition of increasing damping coefficients of the fabric - warp system. During a single movement of the pusher, the peak current value does not change, but the mean value increases.

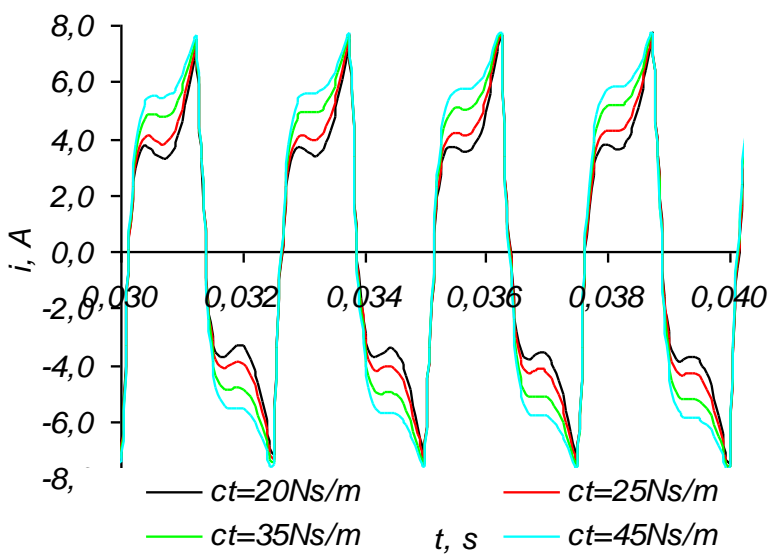

Fig. 11 Waveforms of current intensity against time under different damping coefficients of the fabric - warp system $\left(u= \pm 10 \mathrm{~V} ; k_{t}=40 \mathrm{kN} / \mathrm{m}\right)$

Fig. 12 shows the waveforms of current intensity in the exciter coils when they are supplied with alternating 
voltage of different values. Based on literature data $[8,15]$ it can be concluded, that regarding the analyzed values of voltage, peak values of the flowing current do not exceed levels which are dangerous from the point of view of overheating.

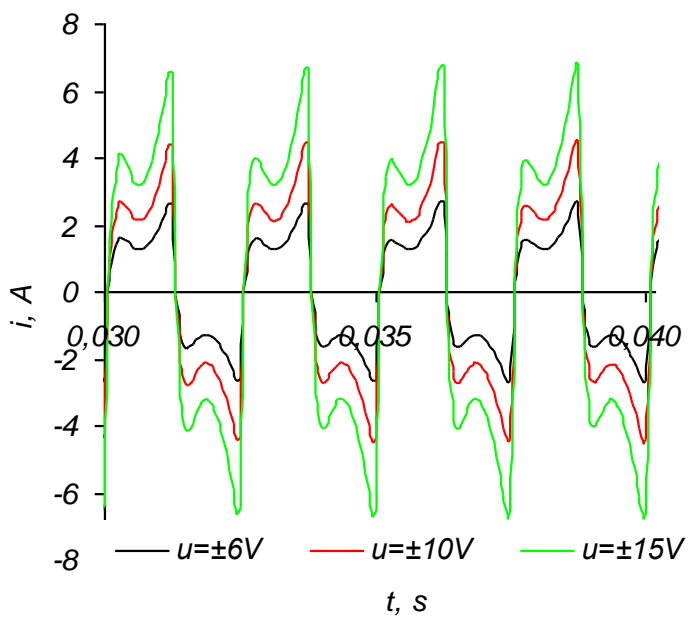

Fig. 12 Waveforms of current intensity against time, for the coils supplied with alternating voltage of different values $\left(k_{t}=40 \mathrm{kN} / \mathrm{m} ; c_{t}=22 \mathrm{Ns} / \mathrm{m}\right)$

In case of the increasing level of the periodically alternating voltage supplied to the exciter coils, the operating amplitude of the pusher vibrating movement increases proportionally. In case of increasing stiffness and damping coefficient of the fabric - warp system, the operating amplitude is reduced. The analysis results are presented in Fig. 13. The minimum operating amplitude of the pusher movement was referred to the peak start-up amplitude. Stiffness and damping coefficients for the phase of contact between the reed and the fabric fell were referred to their values in the initial start-up phase of the vibrating movement.

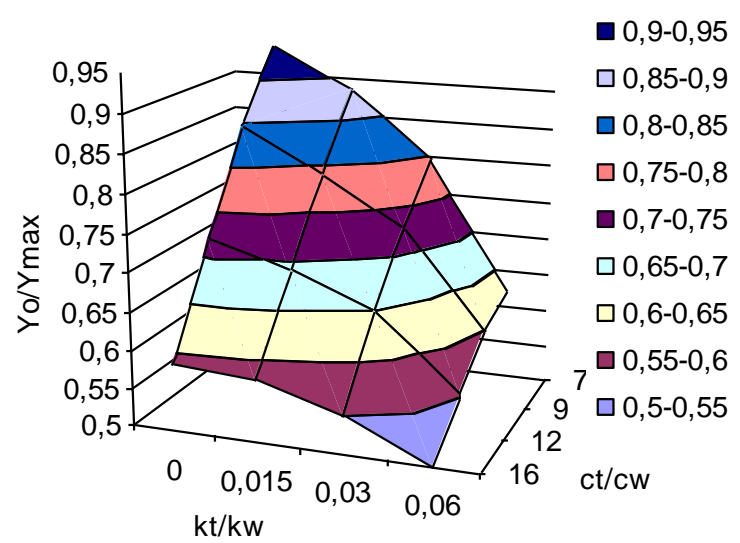

Fig. 13 Relative decrease in the operating amplitude of the pusher vibrating movement as a function of relative damping and stiffness of the fabric - warp system

During thickening of the analyzed fabrics, the operating amplitude of the reed vibrating motion reaches its lowest value of $70-90 \%$ of the initial start-up amplitude. The operating amplitude decrease together with the dynamic parameters of the fabric undergoing thickening. For fabrics characterized by relatively high damping and significant stiffness, the operating amplitude may not reach
$50 \%$ of the start-up one.

\section{Conclusions}

1. For displacements smaller than $1 \mathrm{~mm}$, the inductance of the analyzed exciter coils is almost constant.

2 . The analyzed exciter of the reed movement can be effective in vibrational thickening of the fabrics with typical dynamic parameters, given in the article.

3. The operating amplitudes of the reed vibrating system decrease with the increase in stiffness and damping of the fabric undergoing thickening. For farics with typical dynamic parameters the operating amplitude of the reed vibrating movement can reach $70-90 \%$ of the peak start-up amplitude. For fabrics characterized by relatively high damping and significant stiffness it may not reach $50 \%$ of the start-up amplitude

4. Peak values of current intensity in the colis, exceeding $9 \mathrm{~A}$, are observed during the start-up phase and during the operating movement of the reed in the extreme front position of the slay.

5 . The peak value of the flowing current during the phase of operating vibrating motion of the reed increases together with the stiffness, and the mean value of current increases with the damping coefficient of the thickened fabric.

\section{References}

1. Wroclawski, Z. 1997. The vibration beat up of the weft, FIBRES \& TEXTILES in Eastern Europe 4(19): 78-80.

2. Kossowski, Z.; Kopias, K. 2006. Magnetoelectric driving device for displacements of a guide needle bar in a weaving loom, FIBRES \& TEXTILES in Eastern Europe Vol. 14 2(56): 76-78.

3. Waindok, A. 2008. Computer simulation and verification of measuring the characteristics of the tubular linear motor with permanent magnets, Ph.D. Thesis, University of Opole, Opole, Poland.

4. Katunskis, J. 2004. Theoretical and experimental beatup investigation, FIBRES \& TEXTILES in Eastern Europe Vol. 12, 3(47): 24-28.

5. Azzam, H.A.; Büsgen, A. 2006. Dynamic cloth fell movement. Part I: Critical review, AUTEX Research Journal 6(1): 14-22.

6. Krishnamoorthy, S.G.; Skiedraitè, I. 2015. Development of electromagnetic damper, Mechanika 21(3): 226-233. http://dx.doi.org/10.5755/j01.mech.21.3.9838.

7. Nosek, S. 1994. The dynamics of fabric forming on the loom at high weaving rates, Indian Journal of Fibre \& Textile Research 19: 125-138.

8. Kuchar, M. 2013. The vibration beat-up in the weaving loom. Scientific Bulletin of The Lodz University of Technology, Lodz (In Polish).

9. Tumajer, P.; Ursíny, P.; Bílek, M.; Moučková, E. 2011. Research methods for the dynamic properties of textiles, FIBRES \& TEXTILES in Eastern Europe Vol. 19, 5(88): 33-39.

10. Mikołajezyk, Z. 2011. Optimisation of the knitting process on warp-knitting machines in the aspect of the properties of modified threads and the vibration frequency of the feeding system, FIBRES \& TEXTILES 
in Eastern Europe Vol. 19, 6(89): 75-79.

11. Svetnickienè, V.; Čiukas, R. 2009, Investigation of friction properties of yarns from natural fibres, Mechanika 1(75): 54-58.

12. Svetnickienė, V.; Čiukas, R. 2006. Technical and classical yarns friction properties investigation, Mechanika 4(60): 54-58.

13. Penava, Ž.; Šimić Penava, D.; Knezić, Ž. 2014. Determination of the elastic constants of plain woven fabrics by a tensile test in various directions, FIBRES \& TEXTILES in Eastern Europe Vol. 22, 2(104): 57-63.

14. Yildiz, K.; Şenyürek, V.Y.; Yildiz, Z.; Özen, M.S. 2014. A new approach to the determination of warpweft densities in textile fabrics by using an image proessing technique, Journal of Engineered Fibers and Fabrics 9: 1-5.

15. Kossowski, Z. 2001. Study on the forced electromagnetically valve motion in the valve train, $\mathrm{PhD}$ Thesis, Lodz University of Technology, Lodz, Poland (In Polish).
M. Kuchar, K. Siczek

\section{SIMULATION OF A VIBRATING REED EXCITER \\ FOR THICKENING DIFFERENT FABRICS IN WEAVING LOOM}

\section{S u m m a r y}

The paper presents a vibrating slay mechanism with a flexible weaving reed set into vibrating motion by a special electromagnetic exciter with movable coils segments. The aim of the study was to evaluate the operation of the exciter during vibrational thickening of a fabric. The exciter was analysed in terms of the peak values of current intensity in the coils and the working amplitudes obtained from the vibrating reed. Using Finite Element Method, an axi-symmetrical model of the exciter was elaborated for calculating the changes in the inductance of the coils segments, together with the movement relative to the stator and the current flowing in the coil windings. The obtained results allowed to assume a constant value of coil inductance for the analyzed range of coil displacements. A simulation of the vibrating movement of the reed was carried out while thickening fabrics of different dynamical parameters and supply with periodilally-constant voltage. On the basis of simulations the values of dynamical and electrical parameters were determined. It has been demonstrated that the exciter can be applied in the analysed slay mechanism. The relative decrease in the working amplitude together with the values of the dynamic parameters of the fabric were also determined.

Keywords: permanent magnets, vibration exciters, damping, stiffness, dynamic analysis.

Received October 07, 2015 Accepted September 15, 2016 